\title{
Lidil
}

Revue de linguistique et de didactique des langues

$62 \mid 2020$

Recherches actuelles en didactique du lexique: avancées, réflexions, méthodes

\section{Paul Cappeau et Marie-Noëlle Roubaud, Regards linguistiques sur les textes d'élèves (de 5 à 12 ans)}

Presses universitaires Blaise-Pascal, 2018, 370 p.

Marie-Paule Jacques

\section{OpenEdition \\ Journals}

Édition électronique

URL : http://journals.openedition.org/lidil/8237

DOI : $10.4000 /$ lidil.8237

ISSN : $1960-6052$

Éditeur

UGA Éditions/Université Grenoble Alpes

Édition imprimée

ISBN : 978-2-37747-226-0

ISSN : $1146-6480$

Référence électronique

Marie-Paule Jacques, «Paul Cappeau et Marie-Noëlle Roubaud, Regards linguistiques sur les textes d'élèves (de 5 à 12 ans) », Lidil [En ligne], 62 | 2020, mis en ligne le 03 novembre 2020, consulté le 05 novembre 2020. URL : http://journals.openedition.org/lidil/8237 ; DOI : https://doi.org/10.4000/lidil. 8237

Ce document a été généré automatiquement le 5 novembre 2020.

(C) Lidil 


\section{Paul Cappeau et Marie-Noëlle Roubaud, Regards linguistiques sur les textes d'élèves (de 5 à 12 ans)}

Presses universitaires Blaise-Pascal, 2018, 370 p. Marie-Paule Jacques

\section{RÉFÉRENCE}

Paul Cappeau et Marie-Noëlle Roubaud, Regards linguistiques sur les textes d'élèves (de 5 à 12 ans), Presses universitaires Blaise-Pascal, 2018, 370 p.

1 Lors d'une conférence pédagogique sur l'enseignement de la production d'écrits, je projetai un texte d'écolier assez bancal en demandant à l'auditoire de réfléchir aux conseils qui permettraient à l'élève d'améliorer cet écrit et de façon plus générale de progresser en matière de rédaction. Précaution avait été prise de corriger l'orthographe pour aider les participant-e-s à se dégager des aspects formels, car on sait que l'orthographe est la première cible des commentaires enseignants sur les copies (Pilorgé, 2010). Malgré tout, la difficulté d'entrer véritablement dans le texte de l'élève subsiste, le principal conseil formulé ayant été de « recommencer son texte ».

2 L'œil de l'enseignant doit être éduqué à voir et à comprendre ce que les défaillances dans les textes d'élèves révèlent de la complexité de la tâche qui est celle d'écrire. Ces textes offrent de ce fait un observatoire incomparable pour déterminer les différentes habiletés à construire dans l'objectif de " [s'exprimer] à l'écrit pour raconter, décrire, expliquer ou argumenter de façon claire et organisée»(p.3), qui est l'une des compétences visées par le Socle commun de connaissances, de compétences et de culture.

3 Paul Cappeau et Marie-Noëlle Roubaud l'avaient déjà compris en 2005 en proposant d'«enseigner les outils de la langue avec les productions d'élèves", les auteurs poursuivent ici en exposant une série de "regards linguistiques» qui doivent permettre de mieux appréhender les textes d'élèves et d'en déduire des axes 
d'enseignement appropriés. Leur ouvrage, destiné aux enseignants, qu'ils soient en poste ou en formation, prend largement appui sur les textes d'élèves de 5 à 12 ans pour expliciter et illustrer les diverses dimensions linguistiques impliquées par la production de textes. Chaque chapitre s'attache au décryptage d'une dimension, avec une structuration récurrente qui participe grandement à la lisibilité générale de l'ouvrage et qui en fait un outil de travail efficient au quotidien, en permettant de retrouver aisément chaque rubrique. En ouverture de chapitre, quelques pages de points de repère familiarisent les lecteurs avec la notion travaillée, et cernent ses enjeux pour l'écriture ainsi que les obstacles potentiels pour les élèves. La deuxième partie du chapitre fait fonctionner l'appareil notionnel préalablement introduit à travers l'analyse proprement dite de textes choisis pour mettre en évidence les réussites et les besoins des élèves. Insistons, car elle le mérite, sur cette capacité à aider les allers-retours entre des notions linguistiques générales et la lecture de textes d'élèves. C'est en effet souvent l'opérationnalisation de connaissances «théoriques " qui fait défaut aux enseignants, comme l'a notamment montré Rondelli (2010) en mettant en évidence la connaissance par les enseignants de primaire de certaines recherches en linguistique textuelle, sans que cette connaissance leur donne d'outils pour l'interprétation des écrits des élèves. Après ces analyses détaillées, P. Cappeau et M.-N. Roubaud dressent un bilan des observations menées avant d'offrir dans une $4^{\mathrm{e}}$ partie du chapitre des pistes pour prolonger la réflexion, sous forme d'indications bibliographiques précises.

4 À cette structure qui permet au lecteur un parcours aisé de chacun des onze chapitres, s'ajoute, de par le découpage même de l'ouvrage, une structuration des aspects linguistiques impliqués. Chaque chapitre concerne en effet une dimension de l'écrit: par exemple le codage des sons, la désignation des personnages, les dialogues, l'emploi des temps, la gestion de la grammaire, le lexique, la cohérence des textes; les lecteurs peuvent ainsi comprendre comment dissocier ces aspects et comment appréhender les performances et les besoins des élèves. Tel élève échouant à maitriser convenablement les formes syntaxiques ou la fameuse "concordance des temps" peut néanmoins manifester une excellente gestion des personnages et du fil du texte. Il est indispensable de permettre ces distinctions pour que puissent ensuite être proposés des enseignements à même de faire progresser les élèves sur les aspects moins maitrisés. Or, il a été rappelé en préambule d'une part, que le regard enseignant s'arrête volontiers aux erreurs formelles et peine à voir les autres dimensions, d'autre part que l'ampleur des difficultés, en particulier des élèves les plus jeunes qui ont tout à construire de la maitrise de la textualité, laisse leurs enseignants très démunis. C'est donc œuvre encore une fois particulièrement utile qu'accomplit ce livre, construit pour être un outil au service des enseignants, actuels et futurs. 


\section{AUTEURS}

\section{MARIE-PAULE JACQUES}

LIDILEM (EA 609), Université Grenoble Alpes 